\title{
On establishing qualitative theory to nonlinear boundary value problem of fractional differential equations
}

\author{
Amjad Ali $^{1} \cdot$ Nabeela Khan ${ }^{1} \cdot$ Seema Israr ${ }^{1}$
}

Received: 21 June 2020 / Accepted: 4 February 2021 / Published online: 17 March 2021

(c) The Author(s) 2021

\begin{abstract}
In this article, we study a class of nonlinear fractional differential equation for the existence and uniqueness of a positive solution and the Hyers-Ulam-type stability. To proceed this work, we utilize the tools of fixed point theory and nonlinear analysis to investigate the concern theory. We convert fractional differential equation into an integral alternative form with the help of the Greens function. Using the desired function, we studied the existence of a positive solution and uniqueness for proposed class of fractional differential equation. In next section of this work, the author presents stability analysis for considered problem and developed the conditions for Ulam's type stabilities. Furthermore, we also provided two examples to illustrate our main work.
\end{abstract}

Keywords Arbitrary order differential equations · Topological degree theory · Condensing mapping · Existence results · Stability analysis

Mathematics Subject Classification $26 \mathrm{~A} 33 \cdot 34 \mathrm{~A} 08 \cdot 35 \mathrm{~A} 08$

\section{Introduction}

Fractional calculus is known as the generalization of traditional calculus. In the last few decades, the aforesaid field attended more attention of researchers due to its variety of applications in diverse field of social science and physical science, like physics, chemistry, economics and mechanics. One of the important aspects of aforementioned field that attended the attention of large number of researchers has existence of the solution for boundary value problems (BVPs) of fractional differential equations (FDEs). FDEs are widely applicable in image and signal processing, control theory, model identification, optimization theory, optics, fitting of experimental data for further detail; we refer [1-8] to the readers. Furthermore, some other important applications

Amjad Ali

amjadalimna@yahoo.com

Nabeela Khan

knabeela301@gmail.com

Seema Israr

seemaisrar@gmail.com

1 Department of Mathematics, Govt P.G Jahanzeb College, Saidu Sharif, Swat, Khyber Pakhtunkhwa, Pakistan of FDE are found in diverts fields of engineering, such as fluid dynamic like statistical, electromagnetic, statistical mechanics, fluid flow, polarization, colored noise, solid mechanics, traffic model, colored noise, processes, diffusion, economics and bioengineering see [9-18], in references.

The researchers used tools of fixed point theory and nonlinear analysis to explore the theory up-large extend; for more detail we refer the readers [19-23]. The area devoted to study boundary value problems via topological degree theory for classical order differential equations has been well studied by researches and published number of articles and books. Meanwhile, for fractional differential equations the concern area is quite new and very few papers are available on it. However, the conditions for existence of solution of FDEs, in some articles, need that the operator must be compactness, which restrict the concerned area of research to some specific limitations. Recently, the researchers are interested in some weaker conditions for compactness of the operator. In order to resolve the aforesaid problem, Mawhin [24] used the tools of topological degree theory, to develop the necessary conditions for existence of solution for BVPs of FDEs and IEs. Furthermore, Isais [25] used the degree theory to establish some useful conditions for existence of solutions of FDEs. Recently, Wang et al. [26] used 
the techniques of topological degree theory to develop the conditions for existence of the following non-local Cauchy problem given by

$D^{\varsigma} v(t)=f(t, v(t)), \quad t \in J=[0, T]$,

$v(0)-v_{0}=g(v)$,

where $D^{\varsigma}$ represents the Caputo fractional order derivative, $v_{0} \in \mathbb{R}$ and $f: J \times \mathbb{R} \rightarrow \mathbb{R}$ is continuous function. Furthermore, Ali and Khan [27] study the following BVPs of FDEs with non-local boundary conditions involving fractional integral which is given by

$$
\begin{aligned}
{ }^{c} D^{\varsigma} v(t) & =f(t, v(t)), \quad t \in J=[0,1], \\
v(0) & =g(v), \quad v(1)=\frac{\delta}{\Gamma(q)} \int_{0}^{T}(t-s)^{q-1} v(s) \mathrm{d} s,
\end{aligned}
$$

where ${ }^{c} D^{\varsigma}$ represents Caputo fractional derivatives and $g(v)$ is non-local function, $f: J \times \mathbb{R} \rightarrow \mathbb{R}$ is continuous function.

Another important aspect of concerned theory, which attracted the attention of researchers, has the area devoted of stability analysis of BVPs of FDEs. There are various types of stabilities present in the literature of fractional calculus. One of an important type of stability known as Ulam's stability was initiated by Ulam in (1940). Ulam [28] proposed a question that "Under what conditions does there exists an additive mapping near an approximately additive mapping?". In response to his question Hyers $[29,30]$ replied that "additive mapping in complete norm spaces". Latter on it was tract out is a type of stability as so-called Hyers-Ulam stability. The proposed type of stability was well investigated conventional derivatives by researches. However, for fractional differential equations the concerned type of stability was very rarely investigated and needed more attention of researches to furnish the theory further.

There are various types of stabilities present in the literature for fractional differential equations and integral equations, such as Lyapunov stability [31], asymptotic stability [32], exponential stability [33, 34] and many more, but best to our knowledge one of the interesting type of stability that was origination by Ulam and Hyres commonly known as Hyres-Ulam stability. Rassias [35] initiated a particular kind of stability which is known as Generalized Hyers-Ulam-Rassias stability. Obloza [36] was the author who investigates the concerned stability for DEs. However, the concerned stability was studied for traditional differential equations. Furthermore, for FDEs the area concerning to the stability analysis was at its initial stages and a very few articles had been published, we refer [37], in the references therein. Inspired form the aforementioned importance of the concerned area of research, we consider the following fractional order boundary value problem given by
$D^{\varsigma} v(t)=f(t, v(t)), \quad t \in J=[0,1]$

$v(0)=D^{1} v(0)=D^{2} v(0)=0, \quad v(1)=h(v)$,

where $3<\varsigma \leq 4$ and $\forall s, t \in A C^{4}[0,1], f: J \times \mathbb{R} \rightarrow \mathbb{R}$ is continues and $h(1)=v$ is non-local function. In this work, authors used the tools of topological degree theory and nonlinear analysis to establish necessary conditions for existence solutions and stability analysis for our considered problem. In order to justify the desired results, we provide two examples in last section of the work.

\section{Auxiliary results and definitions}

This section of research work is committed to some fundamental definitions and results of fractional calculus, which are necessary for further correspondence in this work. For more detail (see $[5-8,17,18]$ ).

Definition 1 For all $\varsigma>0$, Gamma function is usually represented by $\Gamma(\varsigma)$ and given by

$\Gamma(\varsigma)=\int_{0}^{1} \mathrm{e}^{-t}(t)^{\varsigma-1} \mathrm{~d} t$.

Definition 2 The fractional order $(\gamma>0)$ integral of a function $u(t): J \rightarrow \mathbb{R}$ is given by

$I^{\gamma} u(t)=\frac{1}{\Gamma(\gamma)} \int_{0}^{t}(t-\sigma)^{\gamma-1} u(\sigma) \mathrm{d} \sigma$,

provided that integral at the right is defined on $(0, \infty)$ point wise.

Definition 3 The famous non-integer order Caputo's function $u(t)$ on any closed interval $[a, b]$ is given by

${ }^{c} D_{0+}^{\gamma} u(t)=\frac{1}{\Gamma(n-\gamma)} \int_{a}^{t}(t-\sigma)^{n-\gamma-1} u^{(n)}(s) \mathrm{d} \sigma$,

where $n=[\gamma]+1$, where $[\gamma]$ is greatest integer less or equal to $\gamma$.

Lemma 1 The solution of fractional order differential equation

${ }^{c} D^{\gamma} u(t)=0, \quad \gamma \in(n-1, n]$,

is given by

$u(t)=u(t)+c_{0}+c_{1} t+c_{2} t^{2}+\cdots+c_{n-1} t^{n-1}$,

where $c_{i} \in \mathbb{R}, \quad$ where $i=0,1,2, \ldots, n$. 
Lemma 2 For FDEs, the following result holds

$I^{\gamma} D^{\gamma} u(t)=u(t)+c_{0}+c_{1} t+c_{2} t^{2}+\cdots+c_{n-1} t^{n-1}$,

for arbitrary $c_{i} \in \mathbb{R}$, where $i=0,1,2, \ldots, n$.

Definition 4 Let us define

$\mathbb{X}=\left\{u(t) \in C J: \quad\|u\|=\max _{t \in[0,1]}|u(t)|\right\}$,

then $(\mathbb{X},\|u\|)$ is a Banach Space.

Definition 5 Let $T: V \rightarrow U$ be a mapping, which is bounded and continuous. Then $T$ is $\varsigma$-Lipschitz, if $\exists K \geq 0$, such that

$\varsigma((B)) \leq K \varsigma(B), \quad \forall B \subset V$ bounded

We also recall that $T: V \rightarrow U$ is Lipschitz, if $\exists K>0$, such that

$\|F x-F y\| \leq K\|x-y\|, \quad \forall \quad x, y \in V$,

and $T$ is strict contraction, if $K<1$.

Proposition 1 If $T, G: V \rightarrow U$ are both $\varsigma$-Lipschitz mapping with constant $K$ and $K^{\prime}$, then $T+G: V \rightarrow U$ is also $\varsigma$ -Lipschitz with $K+K^{\prime}$ constant.

Proposition 2 The mapping $T$ is $\varsigma$-Lipschitz, if $T: V \rightarrow \varsigma$ is Lipschitz with constant $K$.

Proposition 3 If $T: V \rightarrow U$ is compact, then $T$ is $\varsigma$-Lipschitz with zero constant.

Theorem 1 Let $E$ be a measurable set and $\left\{f_{n}\right\}$ be a sequence of measurable function such that

$\lim _{n \rightarrow \infty} f_{n}(v)=f(v) \in E$,

and for every $n \in N$,

$\left|f_{n}(v)\right| \leq h(v) \in E$,

where $f$ is integrable on $E$, then

$\lim _{n \rightarrow \infty} \int_{E} f_{n}(v) \mathrm{d} v=\int_{E} f(v) \mathrm{d} v$.

Definition 6 The Banach space $\mathbb{X}$ is compact, if every sequence $\mathbb{S}_{n}$ contained a convergent sub-sequence in $\mathbb{X}$.

Definition 7 A space $\mathbb{X}$, where every Cauchy sequence of elements of $\mathbb{X}$ converges to an element of $\mathbb{X}$ is called a complete space.
Definition 8 If $A \subseteq X$ is relatively compact, if every sequence of $A$ contained a sub-sequence is convergent in it.

Definition 9 The linear operator $T: V \rightarrow U$ continuous at $v_{0}$, if for any $\varepsilon>0, \exists \delta>0$, such that

$\left|T v-T v_{0}\right|<\varepsilon, \quad \forall \quad\left|v-v_{0}\right|<\delta$.

OR

$T$ is continuous, if $v_{n} \rightarrow v$, then

$F v_{n} \rightarrow F v$.

$O R$

$\lim _{n \rightarrow \infty}\left|v_{n}-v\right| \rightarrow 0$,

which implies that

$\lim _{n \rightarrow \infty}\left|F v_{n}-F v\right| \rightarrow 0$.

Definition 10 The linear operator $T: V \rightarrow U$ is said to be uniformly continuous, if for $\varepsilon>0, \exists \delta>0$, such that

$\left|T v-T v_{0}\right|<\varepsilon, \quad \forall \quad\left|v-v_{0}\right|<\delta$.

OR

$T$ is continuous, if $v_{n} \rightarrow v$, then

$F v_{n} \rightarrow F v$.

$O R$

$\lim _{n \rightarrow \infty}\left|v_{n}-v\right| \rightarrow 0$

which implies that

$\lim _{n \rightarrow \infty}\left|F v_{n}-F v\right| \rightarrow 0$.

Definition 11 The linear operator $T: V \rightarrow U$ is said to be uniformly continuous, if for $\varepsilon>0, \exists \delta>0$, such that

$\left|F v-F v^{*}\right|<\varepsilon \quad \forall\left|v-v^{*}\right|<\delta$.

Definition 12 A family $T$ in $C(J, R)$ is called uniformly bounded, if $\exists$ a constant, where $|f(t)|<k$ for all $t \in J$ and $f \in T$. A family $T$ is equi-continuous, if

$\left|f(v)-f\left(v^{*}\right)\right|<\varepsilon \quad$ for all $v, v^{*} \in J$,

with

$\left|v-v^{*}\right|<\delta$

Theorem 2 If a family $T=(f(v))$ in $C(J, R)$ is uniformly bounded and equi-continuous on $J$, then $F$ has a uniformly convergent sub-sequence $\left(f_{n}(v)\right)=1$. Thus a subset $T$ in $C(J, R)$ is relatively compact, iff T equi-continuous and uniformly bounded on $\mathrm{J}$. 
Theorem 3 Let $\mathbb{X}$ be a Banach space and $T: \mathbb{X} \rightarrow \mathbb{X}$ is function, which is completely continuous, then either

(i) $v=\lambda T v$ has a solution, if $\lambda=1$.

OR

(ii) $\{v \in \mathbb{X}: v=\lambda T v$, for $\lambda \in(0,1))\}$ has a solution.

Definition 13 The solution of FDEs is Hyers-Ulam stable, if $\exists \mathbb{K}_{f}>0$ and we can find $\mathbb{L}_{f}>0$, such that for each solution $v(t)$ to the system there exists a unique solution $v^{*}(t)$, such that

$\left|v(t)-v^{*}(t)\right| \leq \mathbb{K}_{f} \mathbb{L}_{f}$.

\section{Qualitative theory}

In this section, authors present the correspondence results for existence theory of proposed PVBs for FDEs involving conventional derivatives on the boundaries. We developed the relation for integral representation of consider problem and construct the Green function corresponding to the developed integral equation. We use the tools of analysis and degree theory to establish the conditions for uniqueness of the solution of underlying problem of FDE.

Theorem 4 If $3<\varsigma \leq 4$ and $\forall \quad \sigma, t \in[0,1]$, then the solution to fractional differential equation subject to the condition involving ordinary derivatives

$D^{\varsigma} v(t)=f(t, v(t)), \quad t \in P=[0,1]$,

$v(0)=D^{1} v(0)=D^{2} v(0)=0, \quad v(1)=h(v)$,

is given by,

$v(t)=t^{3} h(v)+\int_{0}^{1} \mathbb{H}(t, \sigma) \omega(\sigma) \mathrm{d} \sigma$,

where $\mathbb{U}(t, \sigma)$ represents the Green's function and given by,

$\mathbb{U}(t, \sigma)=\frac{1}{\Gamma(\varsigma)} \begin{cases}(t-\sigma)^{\varsigma-1}-t^{3}(1-\sigma)^{\varsigma-1}, & 0 \leq \sigma \leq t \leq 1, \\ -t^{3}(1-\sigma)^{\varsigma-1}, & 0 \leq t \leq \sigma \leq 1 .\end{cases}$

Proof Consider $f(t, v(t))=\omega(t)$, then (4) become,

$D^{\varsigma} v(t)=\omega(t), \quad 3<\varsigma \leq 4$,

$v(0)=D^{1} v(0)=D^{2} v(0)=0, \quad v(1)=h(v)$.

Then in view of Lemma 2, we have

$v(t)=\gamma_{0}+\gamma_{1} t+\gamma_{2} t^{2}+\gamma_{3} t^{3}+I^{\varsigma} \omega(t)$,

By using the boundary conditions $v(0)=0$ in (6), we get $\gamma_{0}=0$.

Therefore, Eq. (6) becomes

$v(t)=\gamma_{1} t+\gamma_{2} t^{2}+\gamma_{3} t^{3}+I^{\varsigma} \omega(t)$.

Now differentiating Eq. (7), w.r.t " $t$ " and using the boundary condition $D^{1} v(0)=0$, so we get

$\gamma_{1}=0$.

Therefore Eq. (7) becomes

$D^{1} v(t)=\gamma_{1}+2 \gamma_{2} t+3 \gamma_{3} t^{2}+I^{\varsigma-1} \omega(t)$.

Again differentiating Eq. (8), w.r.t " $t$ " and using the boundary condition $D^{2} v(0)=0$, we get

$\gamma_{2}=0$.

Now using the boundary conditions $v(1)=h(v)$ and putting the values of $\gamma_{1}, \gamma_{2}, \gamma_{3}$ in Eq. (6), we get

$\gamma_{3}=h(u)-\frac{1}{\Gamma(\varsigma)} \int_{0}^{1}(1-\sigma)^{\varsigma-1} \omega(\sigma) \mathrm{d} \sigma$,

putting these values in Eq. (6), we get

$v(t)=t^{3} h(v)+\frac{1}{\Gamma(\varsigma)} \int_{0}^{1} \mathbb{H}(t, \sigma) \omega(\sigma) \mathrm{d} \sigma$,

where

$$
\begin{aligned}
& \mathbb{\mathbb { }}(t, \sigma)=\frac{1}{\Gamma(\varsigma)} \\
& \begin{cases}(t-\sigma)^{\varsigma-1}-t^{3}(1-\sigma)^{\varsigma-1}, & 0 \leq \sigma \leq t \leq 1, \\
-t^{3}(1-\sigma)^{\varsigma-1}, & 0 \leq t \leq \sigma \leq 1 .\end{cases}
\end{aligned}
$$

In view of the established results for linear BVP (4), which is equivalent to the following integral equation as

$v(t)=t^{3} h(v)+\int_{0}^{1} \mathbb{H}(t, \sigma) f(\sigma, v(\sigma)) \mathrm{d} \sigma$.

The Eq. (11) is integral representation of our proposed problem (4).

Lemma 3 The function $\mathbb{H}(t, \sigma)$ satisfies the following properties:

(i) $\mathbb{M}(t, \sigma)$ is continuous $\forall \quad s, t \in[0,1]$.

(ii) $\max _{t, s \in[0,1]} \mathbb{M}(t, \sigma) \leq \frac{6 \Gamma(\varsigma)}{\Gamma(4+\varsigma)}$. 


$$
\begin{aligned}
\max _{t, s \in[0,1]} \mathbb{H}(t, \sigma) & =\mathbb{U}(\sigma, \sigma), \\
\max _{t, s \in[0,1]} \int_{0}^{1} \mathbb{H}(t, \sigma) \mathrm{d} \sigma & =\max _{t, s \in[0,1]} \frac{1}{\Gamma(\varsigma)} \int_{0}^{1} \sigma^{3}(1-\sigma)^{\varsigma-1} \mathrm{~d} \sigma, \\
& =\frac{6 \Gamma(\varsigma)}{\Gamma(4+\varsigma)} .
\end{aligned}
$$

Equation (12) is desired value of constructed Green function.

\section{Existence, uniqueness and data dependence results}

In this subsection, we produced some results for existence, uniqueness and data dependence. We also provide the following assumption must hold, which are needed for further investigation in this work.

$\left(H_{1}\right) \quad$ For arbitrary $v, u \in X, \exists$ a constant $\mathbb{K}_{h} \in[0,1)$, such that

$|h(v)-h(u)| \leq \mathbb{K}_{h}\|v-u\|$

$\left(H_{2}\right)$ For arbitrary $v \in X$, there exist $\mathbb{C}_{h}, \mathbb{M}_{h}>0, b_{1} \in[0,1)$, such that

$|h(v)| \leq \mathbb{C}_{h}\|v\|^{b_{1}}+\mathbb{M}_{h}$

$\left(H_{3}\right) \quad$ For arbitrary $(t, v) \in X, \exists \mathbb{C}_{f}, \mathbb{M}_{f}>0, \quad b_{2} \in[0,1)$, such that

$|f(t, v)| \leq \mathbb{C}_{f}\|v\|^{b_{2}}+\mathbb{M}_{f}$.

$\left(H_{4}\right) \quad$ To derive uniqueness of solution the following assumption holds true for $\mathbb{L}_{f}>0$, such that is

$\left|f(t, v)-f\left(t, v^{*}\right)\right| \leq \mathbb{L}_{f}\left\|v-v^{*}\right\|$.

\section{Operator equations}

In this subsection, we convert our obtained integral equation into operator equation. For which we define $T: C(P \times \mathbb{R}, \mathbb{R}) \rightarrow C(P \times \mathbb{R}, \mathbb{R})$,

$T(v)=v, \quad v \in X$,

where

$T v=F v+G v$,

$F: C(P \times \mathbb{R}, \mathbb{R}) \rightarrow C(P \times \mathbb{R}, \mathbb{R})$,

and

$G: C(P \times \mathbb{R}, \mathbb{R}) \rightarrow C(P \times \mathbb{R}, \mathbb{R})$.

Where
$F v=t^{3} h(v)$

$G v=\int_{0}^{1} \mathbb{H}(t, \sigma) f(\sigma, v(\sigma)) \mathrm{d} \sigma$.

Hence the proposed problem gained the operator equation $T v=F v+G v=v$. The fixed points of the constructed operator equation are the desired solutions of concerned BVP (4).

Theorem 5 The operator $F: C(P \times \mathbb{R}, \mathbb{R}) \rightarrow C(P \times \mathbb{R}, \mathbb{R})$ is Lipschitz with constant $\mathbb{C}_{h}<1$ and satisfies the condition

$\|F v\| \leq \mathbb{C}_{h}\|v\|^{b_{1}}+\mathbb{M}_{h}$

Proof We defined $F: C(P \times \mathbb{R}, \mathbb{R}) \rightarrow C(P \times \mathbb{R}, \mathbb{R})$ is given by

$F v=t^{3} h(v)$

To prove $F$ is Lipschitz, we have

$\|F v-F u\|=\max _{t \in[0,1]}\left|t^{3} h(v)-t^{3} h(u)\right|$,

using assumption of $\left(H_{1}\right)$, we have

$\|F v-F u\| \leq k\|v-u\|$,

where $k=\mathbb{K}_{h}<1$.

For growth condition, we consider

$\|F v\|=\max _{t \in[0,1]}\left|t^{3} h(v)\right|$

using assumption of $\left(\mathrm{H}_{2}\right)$, we get

$\|F v\| \leq \mathbb{C}_{h}\|v\|^{b_{1}}+\mathbb{M}_{h}$.

The above result shows that $F$ satisfies the Lipschitz condition with constant $\mathbb{C}_{h}$.

Theorem 6 The operator $G: C(P \times R, R) \rightarrow C(P \times R, R)$ is continuous and satisfies the following

$\|G v\| \leq 2\left(\frac{\mathbb{C}_{f}\|v\|^{b_{2}}+\mathbb{M}_{f}}{\Gamma(\varsigma+1)}\right)$,

for every $v \in A C^{4}[0,1]$.

Proof As $G: C(P \times \mathbb{R}, \mathbb{R}) \rightarrow C(P \times \mathbb{R}, \mathbb{R})$ is given as

$G v=\int_{0}^{1} \mathbb{H}(t, \sigma) f(\sigma, v(\sigma)) \mathrm{d} \sigma$,

To prove, that $G$ is continuous, we have to show that,

$\left\|G v_{n}-G v\right\| \rightarrow 0$ as $n \rightarrow \infty$ 
Let $\left\{v_{n}\right\}$ is a sequence in bounded set, such that

$B_{K}=\{\|v\| \leq K: v \in X\}$.

Now as $f$ is continuous, so $f\left(\sigma, v_{n}(\sigma)\right) \rightarrow f(\sigma, v(\sigma))$ as $n \rightarrow \infty$

$$
\begin{gathered}
t^{3}(1-\sigma)^{\varsigma-1}\left|f\left(\sigma, v_{n}\right)-f(\sigma, u)\right| \\
\leq t^{3}(1-\sigma)^{\varsigma-1}\left\{2 \mathbb{C}_{f}+\mathbb{M}_{f}\right\},
\end{gathered}
$$

is also integrable for all $t \in[0,1]$.

By convergent theorem, we have

$$
\begin{gathered}
\int_{0}^{t}(t-\sigma)^{\varsigma-1}\left[f\left(\sigma, v_{n}(\sigma)\right)\right. \\
\quad-f(\sigma, v(\sigma))] \mathrm{d} \sigma \rightarrow 0,
\end{gathered}
$$

and

$$
\begin{aligned}
& t^{3} \int_{0}^{1}(1-\sigma)^{\varsigma-1}\left[f\left(\sigma, v_{n}(\sigma)\right)\right. \\
& \quad-f(\sigma, v(\sigma))] \mathrm{d} \sigma \rightarrow 0 \text { as } n \rightarrow \infty,
\end{aligned}
$$

So

$\left\|G v_{n}-G v\right\| \rightarrow 0$ as $n \rightarrow \infty$.

Hence, $G$ is continuous.

Now to derive growth condition, we do the following

$$
\begin{aligned}
\|G v\|= & \max _{t \in[0,1]} \mid \frac{1}{\Gamma(\varsigma)} \int_{0}^{t}(t-\sigma)^{\varsigma-1} f(\sigma, v(\sigma)) \mathrm{d} \sigma \\
& -\frac{t^{3}}{\Gamma(\varsigma)} \int_{0}^{1}(1-\sigma)^{\varsigma-1} f(\sigma, v(\sigma)) \mathrm{d} \sigma \mid,
\end{aligned}
$$

Hence by assumption $\left(H_{3}\right)$, we get

$$
\begin{aligned}
\|G v\| & \leq \frac{\mathbb{C}_{f}\|v\|^{b_{2}}+\mathbb{M}_{f}}{\Gamma(\alpha+1)}(1+1), \\
& \leq 2\left(\frac{\mathbb{C}_{f}\|v\|^{b_{3}}+\mathbb{M}_{f}}{\Gamma(\varsigma+1)}\right) .
\end{aligned}
$$

Thus $G$ satisfies the defined growth condition.

Theorem 7 The operator $G: C(P \times \mathbb{R}, \mathbb{R}) \rightarrow C(P \times \mathbb{R}, \mathbb{R})$ is Compact and $\alpha$-Lipschitz with constant zero.

Proof As $G: C(P \times \mathbb{R}, \mathbb{R}) \rightarrow C(P \times \mathbb{R}, \mathbb{R})$ is given by

$$
\begin{aligned}
G v= & \frac{1}{\Gamma(\varsigma)}\left(\int_{0}^{t}(t-\sigma)^{\varsigma-1} f\left(\sigma, v_{n}(\sigma)\right) \mathrm{d} \sigma\right. \\
& \left.+t^{3} \int_{0}^{1}(1-\sigma)^{\varsigma-1} f\left(\sigma \cdot v_{n}(\sigma)\right) \mathrm{d} \sigma\right) .
\end{aligned}
$$

In order to prove $G$ compact, we have to show that $G$ is both equi-continuous and uniform bounded.

Let us consider $D \subseteq B_{k} \subseteq X$, for this is sufficient to show that $G(D)$ is relatively compact in X. Let $v_{n}$ in $D \subseteq B_{k}, \quad \forall v_{n} \in D$, in the light of Theorem 6, we have

$\left\|G v_{n}\right\| \leq 2\left(\frac{\mathbb{C}_{f}\|v\|^{b_{2}}+N_{f}}{\Gamma(\varsigma+1)}\right)$.

So $G$ is bounded. Now, for equi-continuous. Consider $0<t<\tau<1$.

$$
\begin{aligned}
G v_{n}(\tau)= & \frac{1}{\Gamma(\varsigma)}\left(\int_{0}^{\tau}(\tau-\sigma)^{\varsigma-1} f\left(\sigma, v_{n}(\sigma)\right) \mathrm{d} \sigma\right. \\
& \left.+\tau^{3} \int_{0}^{1}(1-\sigma)^{\varsigma-1} f\left(\sigma, v_{n}(\sigma)\right) \mathrm{d} \sigma\right), \\
& \left\|G v_{n}(\tau)-G v_{n}(t)\right\| \\
\leq & \mid \frac{1}{\Gamma(\varsigma)}\left(\int_{0}^{\tau}(\tau-\sigma)^{\varsigma-1} f\left(\sigma, v_{n}(\sigma)\right) \mathrm{d} \sigma\right. \\
& \left.-\int_{0}^{t}(t-\sigma)^{\varsigma-1} f\left(\sigma, v_{n}(\sigma)\right) \mathrm{d} \sigma\right) \mid \\
& +\mid \frac{1}{\Gamma(\varsigma)}-\int_{0}^{1}\left(\tau^{3}(1-\sigma)^{\varsigma-1}-t^{3}(1-\sigma)^{\varsigma-1}\right) \\
& {\left[f\left(\sigma, v_{n}(\sigma)\right)\right] \mathrm{d} \sigma \mid, }
\end{aligned}
$$

Now by using assumption $\left(\mathrm{H}_{3}\right)$, we have

$$
\begin{aligned}
\left|G v_{n}(\tau)-G v_{n}(t)\right| \leq & \frac{\mathbb{C}_{f}\|v\|^{b_{2}}+\mathbb{M}_{f}}{\Gamma(\varsigma+1)} \\
& \left(\tau^{\varsigma}-t^{\varsigma}+\left(\tau^{3}-t^{3}\right)^{\varsigma}\right) .
\end{aligned}
$$

As $t \rightarrow \tau$, the R.H.S in above relation tends to 0 , that is

$\left|G u_{n}(\tau)-G u_{n}(t)\right| \rightarrow 0 \quad$ as $t \rightarrow \tau$.

Thus $G u_{n}$ is uniformly continuous.

Thus $\left\{G u_{n}\right\}$ is equi-continuous. Hence $G(D) \subset G(D)$. Thus by Arzela Ascoli theorem $G(D)$ is relatively compact in $X$. Further $G$ is $\alpha$-Lipschitz with constant zero. 


\section{Uniqueness of solutions for BVP (4) of FDE}

In this section, we developed the condition for uniqueness and boundness of BVP (4).

Theorem 8 The consider BVP (4) has at least one solution and the set of the solutions is bounded.

Proof As the operators $F, G, T: C(P \times R, R) \rightarrow C(P \times R, R)$ are continuous and bounded. Further $F, G$ are $\alpha$-Lipschitz with constant $K$ and 0 . The operator $T$ is $\alpha$-Lipschitz with Lipschitz constant $K$. Since $\mathbb{K}_{h}<1$, so $T$ is a contraction mapping.

Consider the set of solution

$S=\{v \in X: 0 \leq \lambda \leq 1, \quad v=\lambda T v\}$ is bounded.

For boundness, consider

$$
\begin{aligned}
\|v\| & =\max _{t \in[0,1]}|\lambda T v| \leq \max _{t \in[0,1]} \lambda|T v| \\
& \leq \max _{t \in[0,1]} \lambda|F v+G v|, \\
\|v\| & \leq \lambda\left(\mathbb{C}_{h}\|v\|^{b_{1}}+\mathbb{M}_{h}+2\left(\frac{\mathbb{C}_{f}\|v\|^{b_{2}}+\mathbb{M}_{f}}{\Gamma(\varsigma+1)}\right)\right) .
\end{aligned}
$$

From above it is clear that $S$ is bounded. If not, let $\varsigma=\|v\| \rightarrow \infty$ as $0<b_{1}, b_{2}<1$,

$\|v\|=\|\lambda T v\| \leq \lambda\|T v\| \leq \lambda\|F v+G v\|$,

$$
1 \leq \frac{\lambda}{\varsigma}\left(\mathbb{C}_{h} \varsigma^{b_{1}}+2\left(\frac{\mathbb{C}_{f} \varsigma^{b_{2}}+\mathbb{M}_{f}}{\Gamma(\varsigma+1)}\right)\right),
$$

as $\varsigma \rightarrow \infty$, which means $1 \leq 0$ is not possible. Hence a set $S$ is bounded.

Theorem 9 If $\delta=K+\frac{2 \mathbb{L}_{f}}{\Gamma(\varsigma+1)} \leq 1$, then our proposed $B V P$ (4) has a unique solution.

Proof Consider $u, v \in X$, such that

$$
\begin{aligned}
& \|T v-T u\|=\max _{t \in[0,1]}|T v(t)-T u(t)|, \\
& =\max _{t \in[0,1]}|F v(t)+G v(t)-F u(t)+G u(t)|, \\
& \leq \max _{t \in[0,1]}|F v(t)-G u(t)|+\max _{t \in[0,1]}|F v(t)+G u(t)|, \\
& \leq K\|v-u\|+\max _{t \in[0,1]} \mid \frac{1}{\Gamma(\varsigma)} \int_{0}^{t}(t-\sigma)^{\varsigma-1}(f(\sigma, v(\sigma)) \mathrm{d} \sigma \\
& \quad+\frac{t^{3}}{\Gamma(\varsigma)} \int_{0}^{1}(1-\sigma)^{\varsigma-1}(f(\sigma, u(\sigma)) \mathrm{d} \sigma \mid,
\end{aligned}
$$

using assumption $\left(H_{4}\right)$, we have
$|T v(t)-T u(t)| \leq\left(K+\frac{2 \mathbb{L}_{f}}{\Gamma(\varsigma+1)}\right)\|v-u\|$,

$|T v(t)-T u(t)|=\Delta\|v-u\| \leq \delta$,

where

$\Delta=K+\frac{2 \mathbb{L}_{f}}{\Gamma(\varsigma+1)}$.

Hence, there exists unique solution to BVP (4).

\section{Stability analysis of BVP of FDEs}

This section of research work, is devoted to stability analysis of consider BVP (4). We developed the condition for the Hyers-Ulam-type stability for proposed BVP of FDE.

Theorem 10 If the assumption $\left(H_{1}\right)-\left(H_{4}\right)$ holds, then the solution is Hyers-Ulam stable.

Proof Let $v$ and $v^{*} \in C^{4}(I, R)$ be any two solution of BVPs (4). For stability

$$
\begin{aligned}
D^{\varsigma} v(t) & =f(t, v(t)), \quad t \in I, \varsigma \in(3,4], \\
v(0) & =D^{1} v(0)=D^{2} v(0)=0, \\
v(1) & =h(v), \\
v(t) & =t^{3} h(v)+\int_{0}^{1} \mathbb{H}(t, \sigma) f(\sigma, v(\sigma)) \mathrm{d} \sigma, \\
v^{*}(t) & =t^{3} h\left(v^{*}\right)+\int_{0}^{1} \mathbb{H}(t, \sigma) f\left(\sigma, v^{*}(\sigma)\right) \mathrm{d} \sigma .
\end{aligned}
$$

Consider

$$
\begin{aligned}
\left|v(t)-v^{*}(t)\right| & =\max _{t \in[0,1]} \mid t^{3} h(v) \\
& \left.+\int_{0}^{1} \mathbb{H}(t, \sigma) f(\sigma, v(\sigma)) \mathrm{d} \sigma\right]-t^{3} h\left(v^{*}\right) \\
& +\int_{0}^{1} \mathbb{H}(t, \sigma) f\left(\sigma, v^{*}(\sigma)\right) \mathrm{d} \sigma \mid, \\
& \leq \max _{t \in[0,1]}\left|t^{3} h(v)-t^{3} h\left(v^{*}\right)\right| \\
& +\max _{t \in[0,1]} \mid \int_{0} \mathbb{H}(t, \sigma)[f(\sigma, v(\sigma) \\
& \left.-f\left(\sigma, v^{*}(\sigma)\right)\right] \mathrm{d} \sigma \mid,
\end{aligned}
$$


using assumption $\left(H_{1}\right)$ and $\left(H_{4}\right)$, we have

$$
\begin{aligned}
\leq & \mathbb{K}_{h}\left\|v-v^{*}\right\| \\
& +\max _{t \in[0,1]} \int_{0}^{1} \mathbb{H}(t, \sigma) \mathrm{d} \sigma \mathbb{L}_{f}\left\|v-v^{*}\right\|,
\end{aligned}
$$

using maximum value of green function

$$
\begin{aligned}
& \left\|v(t)-v^{*}(t)\right\| \leq\left\|v-v^{*}\right\|\left[\mathbb{K}_{h}+\frac{6 \Gamma(\varsigma)}{\Gamma(4+\varsigma)} \mathbb{L}_{f}\right], \\
& \quad\left\|v(t)-v^{*}(t)\right\| \leq K_{1} K_{2}, \\
& \text { where } K_{1}=\left\|v-v^{*}\right\| \text { and } K_{2}=\left[\mathbb{K}_{h}+\frac{6 \Gamma(\varsigma)}{\Gamma(4+\varsigma)} \mathbb{L}_{f}\right] .
\end{aligned}
$$

Hence the solution of BVP 4 is Hyers-Ulam stable.

\section{Examples}

In this section, we provide some examples to illustrate the main work for proposed BVP of FDEs.

Example 1 Consider the following BVP for FDEs

$$
\begin{aligned}
D^{7 / 2} v(t) & =\frac{t^{3} \sin v(t)}{40}, \\
v(0) & =D^{1} v(0)=D^{2} v(0)=0, \\
v(1) & =\frac{\cos v(t)}{30} .
\end{aligned}
$$

where $\varsigma=7 / 2$, Now where $\mathbb{M}(t, \sigma)$ is,

$$
\begin{aligned}
& \mathbb{H}(t, \sigma)=\frac{1}{\Gamma(7 / 2)} \\
& \begin{cases}(t-\sigma)^{5 / 2}-t^{3}(1-\sigma)^{5 / 2}, & 0 \leq \sigma \leq t \leq 1, \\
-t^{3}(1-\sigma)^{5 / 2}, & 0 \leq t \leq \sigma \leq 1 .\end{cases}
\end{aligned}
$$

Now

$|f(t, v)| \leq \mathbb{C}_{f}|v|^{b_{2}}+\mathbb{M}_{f}$

where

$$
\begin{gathered}
C_{f}=1 / 40, \quad M_{f}=1 / 40, \quad b_{2}=1, \\
|f(t, v)| \leq 0.05,
\end{gathered}
$$

After some calculation, we have

$\Delta=K+\frac{2 \mathbb{L}_{f}}{\Gamma(\varsigma+1)}$,

where

$$
\begin{gathered}
K=1 / 30, L_{f}=1 / 40, \varsigma=7 / 2, \\
|T v(t)-T u(t)| \leq 0.0376<1 .
\end{gathered}
$$

Assumption $\left(H_{1}\right)-\left(H_{2}\right)$ holds; therefore solution of concerned problem has at least one solution.

For the stability of BVP (14) of FDE, we have

$\left|v(t)-v^{*}(t)\right| \leq\left\|v-v^{*}\right\|\left[\mathbb{K}_{h}+\frac{6 \Gamma(\varsigma)}{\Gamma(4+\varsigma)} \mathbb{L}_{f}\right]$.

Let

$$
\begin{gathered}
K_{h}=1 / 30, L_{f}=1 / 40 \text { and } \varsigma=7 / 2, \\
\left|v(t)-v^{*}(t)\right| \leq 0.0045<1 .
\end{gathered}
$$

Hence the solution BVP (14) has a unique solution and the solution has stable.

Example 2 Consider the BVP for FDEs

$$
\begin{aligned}
D^{5 / 2} v(t) & =\frac{t+\sin ^{1 / 2} v(t)}{50+\mathrm{e}^{-\pi t}}, \\
v(0) & =D^{1} v(0)=D^{2} v(0)=0, \\
v(1) & =\frac{\cos v(t)}{40} .
\end{aligned}
$$

where $\mathbb{H}(t, \sigma)$ is

$$
\begin{aligned}
& \mathbb{U}(t, \sigma)=\frac{1}{\Gamma(5 / 2)} \\
& \begin{cases}(t-\sigma)^{3 / 2}-t^{3}(1-\sigma)^{3 / 2}, & 0 \leq \sigma \leq t \leq 1, \\
-t^{3}(1-\sigma)^{3 / 2}, & 0 \leq t \leq \sigma \leq 1 .\end{cases}
\end{aligned}
$$

Now

$|f(t, v)| \leq \mathbb{C}_{f}|v|^{b_{2}}+\mathbb{M}_{f}$.

where $C_{f}=1 / 50, M_{f}=1 / 50, b_{2}=1 / 2$,

After some calculation, we have

$\Delta=K+\frac{2 \mathbb{L}_{f}}{\Gamma(\varsigma+1)}$,

where

$$
\begin{gathered}
K=1 / 40, L_{f}=1 / 50, \varsigma=5 / 2, \\
|T v(t)-T u(t)| \leq 0.03074<1 .
\end{gathered}
$$

The assumption $\left(H_{1}\right)-\left(H_{2}\right)$ holds. Hence the proposed BVP is at least one solution.

For the stability of BVP (16) of FDE,

$$
\left|v(t)-v^{*}(t)\right| \leq\left\|v-v^{*}\right\|\left[\mathbb{K}_{h}+\frac{6 \Gamma(\varsigma)}{\Gamma(4+\varsigma)} \mathbb{L}_{f}\right],
$$

Let 


$$
\begin{gathered}
K_{h}=1 / 40, L_{f}=1 / 50 \text { and } \varsigma=5 / 2, \\
\left|v(t)-v^{*}(t)\right| \leq 0.0011097<1 .
\end{gathered}
$$

Hence the solution BVP (16) has a unique solution and the solution has stable.

Open Access This article is licensed under a Creative Commons Attribution 4.0 International License, which permits use, sharing, adaptation, distribution and reproduction in any medium or format, as long as you give appropriate credit to the original author(s) and the source, provide a link to the Creative Commons licence, and indicate if changes were made. The images or other third party material in this article are included in the article's Creative Commons licence, unless indicated otherwise in a credit line to the material. If material is not included in the article's Creative Commons licence and your intended use is not permitted by statutory regulation or exceeds the permitted use, you will need to obtain permission directly from the copyright holder. To view a copy of this licence, visit http://creativecommons.org/licenses/by/4.0/.

\section{References}

1. El-Shahed, M., Nieto, J.J.: Nontrivial solutions for a nonlinear multi-point boundary value problem of fractional order. Comput. Math. Appl. 59(11), 3438-3443 (2010)

2. Agarwal, R., Benchohra, M., Hamani, S.: A survey on existence results for boundary value problem of non-linear fractional differential equations and inclusions. J. Math. Appl. 109(3), 973-1033 (2010)

3. El-Sayad, A.M.: Non-linear functional differential equation of ordinary orders. Nonlinear Anal. 33, 181-186 (1998)

4. Khan, R.A., Shah, K.: Existence and uniqueness of solution to fractional order multi-point boundary value problems. Commun. Appl. Anal. 19, 515-526 (2015)

5. Zhou, L., Hu, G.: Global exponential periodicity and stability of cellular neural networks with variable and distributed delays. Appl. Math. Comput. 195(2), 402-411 (2008)

6. Raja, M., Khan, J.A., Qureshi, I.M.: Solution of fractional order system of Bagley-Torivk equation using evolutionary computational intelligence. Math. Prob. Eng. 2011, 18 (2011)

7. Mohebbi, A., Asgari, Z., Dehghan, M.: Numerical solution of non-linear Jaulent-Miodek and Whitam-Brore-Kaup. Commun. Nonlinear Sci. Numer. Simul. 17(12), 4602-4610 (2012)

8. Goodrich, C.: Existence of a positive solution to a class of fractional differential equations. J. Comput. Math. Appl. 23(9), 10501055 (2010)

9. Caputo, M.: Linear models of dissipation whose Q is almost frequency independent. Int. J. Geogr. Sci. 13(5), 529-539 (1967)

10. Yang, Y., Ma, Y., Wang, L.: Legendre polynomails operational matrix method for solving fractional partial differential equations with variable coefficients. Math. Probl. Eng. 2015, 1-9 (2015)

11. Carpinteri, A., Mainardi, F.: Fractals and Fractional Calculus Continuum Mechanics. Springer, Berlin (1997)

12. Rostamy, D., Karimi, K., Mohamadi, E.: Solving fractional partial differential equations by an efficient new basis. Int. J. Appl. Math. Comput. Sci. 5(1), 6-12 (2013)

13. Tarasov, V.E.: Fractional integro differential equations for electromagnetic waves in dielectric media. Theor. Math. Phys. 158(3), 355-359 (2009)
14. Baillie, R.T.: Long memory processes and fractional integration in econometrics. J. Econ. 73, 5-59 (1996)

15. Magin, R.L.: Fractional calculus in bioengineering. Crit. Rev. Biomed. Eng. 32(2), 1-104 (2004)

16. Magin, R.L.: Fractional calculus in bioengineering-part 2. Crit. Rev. Biomed. Eng. 32(2), 105-193 (2004)

17. Magin, R.L.: Fractional calculus in bioengineering-part 3. Crit. Rev. Biomed. Eng. 32(3/4), 194-377 (2004)

18. Lacroix, S.F.: Calcul Differentiel et du. Trait.6 du Calc. Integ. Paris 3, 409-410 (1819)

19. Abbas, M.I.: Existence and uniqueness of solution for a boundary value problem of fractional order involving two Caputo's fractional derivatives. Adv. Differ. Equ. 2015, 252 (2015)

20. Ahmad, B., Nieto, J.J.: Existence results for a coupled system of nonlinear fractional differential equations with three-point boundary conditions. Comput. Math. Appl. 58(2009), 1838-1843 (2009)

21. Kumam, P., et al.: Existence results and Hyers-Ulam stability to a class of nonlinear arbitrary order differential equations. J. Nonlinear Sci. Appl. 10(2017), 2986-2997 (2017)

22. Ali, G., et al.: On existence and stability results to a class of boundary value problems under Mittag-Leffler power law. Adv. Differ. Equ. 2020(407), 1-13 (2020)

23. Ali, Z., et al.: Modeling and analysis of novel COVID-19 under fractal-fractional derivative with Case Study of Malaysia. Fractals (2020). https://doi.org/10.1142/S0218348X21500201

24. Mawhin, J.: Topological Degree Methods in Nonlinear Boundary Value Problems, CMBS Regional Conference Series in Mathematics, vol. 40. American Mathematical Society, Providence (1979)

25. Isaia, F.: On a nonlinear integral equation without compactness. Acta Math. Univ. Comen. 75(2), 233-240 (2016)

26. Wang, J., Zhou, Y., Wei, W.: Study in fractional differential equations by means of topological degree methods. Num. Funct. Anal. Optim. 33(2), 216-238 (2012)

27. Ali, A., Khan, R.A.: Existence of solutions of fractional differential equations via topological degree theory. J. Comput. Theor. Nanosci. 13(2016), 1-5 (2016)

28. Ulam, S.M.: Problems in Modern Mathematic. Wiley, New York (1964)

29. Hyers, D.H.: On the stability of the linear functional equation. Natl. Acad. Sci. U. S. A. 27(4), 222-224 (1941)

30. Aoki, T.: On the stability of the linear transformation in Banach space. J. Math. Soc. Jpn. 2, 64-66 (1950)

31. Trigeassou, J., et al.: A Lyapunov approach to the stability of fractional differential equations. Signal Process. 91(3), 437-445 (2011)

32. Agarwal, R., Hristova, S., Q'Regan, D.: Stability of solutions to impulsive caputo fractional differential equation. Electron. J. Differ. Equ. 2016, 1-22 (2016)

33. Agarwal, R., O'Regan, D., Hristova, S.: Stability of Caputo fractional differential equations by Lyapunov function. Appl. Math. 60, 653-676 (2015)

34. Rassias, T.M.: On the stability of the linear mapping in Banach space. Proc. Am. Math. Soc. 72(2), 297-300 (1978)

35. Obloza, M.: Hyers stability of the linear differential equation. Prace Math. 13(13), 259-270 (1993)

36. Wang, J., Lv, L., Zhou, Y.: Ulam stability and data dependence for fractional differential equations with Caputo derivative. Electron. J. Qual. Theory Differ. Equ. 2011, 1-10 (2011)

37. Gao, Z., Yu, X., Wang, J.: Exp-type Ulam-Hyers stability of fractional differential equations with positive constant coefficient. Adv. Differ. Equ. 238(2015), 1-10 (2015) 